Article

\title{
Six-Month Predictive Value of Diuretic Resistance Formulas in Discharged Heart Failure Patients after an Acute Decompensation
}

\author{
Mauro Feola ${ }^{1, *}$, Arianna Rossi ${ }^{2}$, Marzia Testa ${ }^{1}$, Cinzia Ferreri ${ }^{1}$, Alberto Palazzuoli ${ }^{3}$, \\ Guido Pastorini ${ }^{1}$ and Gaetano Ruocco ${ }^{1}$ \\ 1 Cardiology Division, Ospedale Regina Montis Regalis, Mondovi' ASL CN1, 12084 Cuneo, Italy; \\ marzia.testa@aslcn1.it (M.T.); cinzia.ferreri@aslcn1.it (C.F.); guido.pastorini@aslcn1.it (G.P.); \\ gaetanomaria.ruocco@aslcn1.it (G.R.) \\ 2 School of Geriatry, Universita' degli Studi Torino, 10124 Torino, Italy; arirossi_ary@hotmail.com \\ 3 Cardiovascular Diseases Unit, Ospedale Le Scotte Universita' Siena, 53100 Siena, Italy; palazzuoli2@unisi.it \\ * Correspondence: m_feola@virgilio.it; Fax: +39-0174677306
}

Received: 28 July 2020; Accepted: 9 September 2020; Published: 11 September 2020

\begin{abstract}
Background. The diuretic response has been shown to be a robust independent marker of cardiovascular outcomes in acute heart failure patients. The objectives of this clinical research are to analyze two different formulas (diuretic response (DR) or response to diuretic (R-to-D)) in predicting 6-month clinical outcomes. Methods: Consecutive patients discharged alive after an acute decompensated heart failure (ADHF) were enrolled. All patients underwent N-terminal-pro hormone BNP (NT-proBNP) and an echocardiogram together with DR and R-to-D calculation during diuretic administration. Death by any cause, cardiac transplantation and worsening heart failure (HF) requiring readmission to hospital were considered cardiovascular events. Results: 263 patients (62\% male, age 78 years) were analyzed at 6-month follow-up. During the follow-up 58 (22.05\%) events were scheduled. Patients who experienced CV-event had a worse renal function $(p=0.001)$, a higher NT-proBNP $(p=0.001)$, a lower left ventricular ejection fraction $(p=0.01)$, DR $(p=0.02)$ and R-to-D ( $p=0.03)$. Spearman rho's correlation coefficient showed a strong direct correlation between $\mathrm{DR}$ and $\mathrm{R}$ to $\mathrm{D}$ in all patients $(\mathrm{r}=0.93 ; p<0.001)$ and both in heart failure with reduced ejection fraction (HFrEF) $(\mathrm{r}=0.94 ; p<0.001)$ and HF preserved ejection fraction (HFpEF) $(\mathrm{r}=0.91 ; p<0.001)$. At multivariate analysis, a value of R-to-D $<1.69 \mathrm{~kg} / 40 \mathrm{mg}$, but only $<0.67 \mathrm{~kg} / 40 \mathrm{mg}$ for DR were significantly related to poor 6-month outcome ( $p=0.04$ and $p=0.05$, respectively). Receiver operating characteristic (ROC) curve analyses demonstrated that DR and R-to-D are equivalent in predicting prognosis (area under curve (AUC): 0.39 and 0.40, respectively). Only R-to-D was inversely related to in-hospital stay $(\mathrm{r}=-0.23 ; p=0.01)$. Conclusion: Adding diuresis to DR seemed to provide a better risk assessment in alive HF patients discharged after an acute decompensation.
\end{abstract}

Keywords: NT-proBNP; prognosis; heart failure; diuretic resistance

\section{Introduction}

In heart failure (HF) patients the evaluation of prognosis is currently performed on multiple clinical and laboratory variables, but it remains unpredictable because of sudden hemodynamic deterioration related to cardiac and extracardiac reasons and the lack of consistent universally validated algorithm [1]. The presence of renal dysfunction and arterial hypotension may stratify patients with the worst clinical outcome after discharging for an acute decompensated heart failure episode [2]. A single evaluation of brain natriuretic peptide (BNP) plasma level proved to be a robust risk stratification method being a sensitive diagnostic marker of left ventricular dysfunction [3,4] and predicting cardiovascular events in 
HF patients [5-8]. The fluid overload seemed to be crucial for several clinical features which characterize the whole spectrum of congestive heart failure (CHF). Loop diuretics are a cornerstone of CHF therapy, but the question of whether high dosage of diuretics may cause "per se" a worsening clinical prognosis remained unanswered [9]. Diuretic resistance has been widely defined as an impaired sensitivity to diuretics resulting in reduced natriuresis and diuresis limiting the obtaining of euvolemia [10]. In acute decompensation, the development of worsening renal function, called "cardiorenal syndrome", may be a limitation in achieving an adequate volume removal [11]. If an effective decongestion is not achieved, a modest decline in renal function (calculated by serum creatinine plasma level elevation from to 0.3 to $0.5 \mathrm{mg} / \mathrm{dL}$ ) has been associated with longer hospitalizations and increases mortality rate [12,13]. Furthermore, other clinical experiences $[14,15]$ concluded that a transient decline in renal function during diuretic therapy in acute decompensation should not be interpreted as an unfavorable event. For instance, the last grade of worsening renal function ( $<30$ days or longer) seemed to influence the mortality risk (hazard ratio (HR) from 0.8 to 3.2) [14] and that the worst prognosis has been observed in those who developed an increase of creatinine plasma level $>0.1 \mathrm{mg} / \mathrm{dL}$ per day in the first week. Despite the clinical importance of this issue, the definition of "diuretic resistance" has long remained empirical and not quantitative, not measurable. Valente et al. [16], using the measurement of the weight change on Day 4 per $40 \mathrm{mg}$ of furosemide on Days 1-3 (equivalent doses: bumetanide $1 \mathrm{mg}$; torasemide $20 \mathrm{mg}$ ), calculated the "diuretic response" (DR), considered to be the first clinical experience to give a quantitative measurement of diuretic resistance. A worse diuretic response (the lowest quintiles corresponded to the worst prognosis) was independently correlated with 180-day mortality (HR 1.42), 60-day death or renal or cardiovascular rehospitalization (HR 1.34) and 60-day rehospitalization (HR 1.57). These data represented a robust indicator that a blunted loss in weight in CHF patients admitted for acute decompensation described a "diuretic resistance".

More recently, a formula including diuresis on the fourth day of diuretic treatment, demonstrated to predict cardiovascular outcomes in a single-center clinical experience (R-to-D $\leq 1.2 \mathrm{~kg} / 40 \mathrm{mg}$ furosemide $p=0.009, \log$ rank 10.96) [17].

This clinical research aims to verify if the inclusion of diuresis in the formula for diuretic response stratified prognosis in CHF patient treated with loop diuretics during an acute episode of cardiac decompensation (RESEARCH REGISTRY1965) at a short-term follow-up.

\section{Methods}

All consecutive HF subjects discharged alive after an acute episode of cardiac decompensation with fluid overload (clinically or according to water composition) were enrolled in an out-patient clinic follow-up (from January 2017 to December 2019). Patients were classified as having CHF according to the criteria commonly accepted in literature [18], such as the presence of 2 major criteria or 1 major criterion +2 minor criteria according to the Framingham score and a NYHA functional class II, III or $\mathrm{IV}$, due to an exacerbation of symptoms with at least 1 class deterioration. Patients with symptoms of CHF, plasma NT-proBNP $>125 \mathrm{pg} / \mathrm{mL}$ and left ventricular ejection fraction (LVEF) $<50 \%$ were defined as both in heart failure with reduced ejection fraction (HFrEF). Patients with symptoms of CHF, plasma NT-proBNP $>125 \mathrm{pg} / \mathrm{mL}, \mathrm{LVEF}>50 \%$ and diastolic dysfunction were defined as HF preserved ejection fraction (HFpEF). The presence of inadequate echo images or no adherence to the therapy (medication adherence was assessed using a self-reported measure) and disagreement with the periodical follow-up were considered exclusion criteria. Eligible patients underwent a clinical examination, a 12-lead electrocardiogram, BNP plasma level determination, body weight measurement at admission and on Day 4 of hospitalization, water composition (on admission and at discharge), 6-min walk test (6MWT), noninvasive cardiac output and a transthoracic echocardiogram within $48 \mathrm{~h}$ of hospital discharge. Serum creatinine was checked on clinical stability and glomerular filtration rate 
(GFR) calculated with the Chronic Kidney Disease-Epidemiology Collaboration (CKD-EPI) equation. The measurement to "diuretic response", as described by Valente et al. [16], was calculated as follows:

$$
\mathrm{DR}=\left[\left(\mathrm{W}_{\mathrm{d} 4}-\mathrm{W}_{\text {baseline }}\right) / \text { Fdose }\right]
$$

where $\mathrm{W}_{\mathrm{d} 4}$ is the weight at day 4 (in $\mathrm{kg}$ ), $\mathrm{W}_{\text {baseline }}$ is the weight at baseline, Fdose is the dose of furosemide on days 1-3 (40 mg) (equivalent doses: bumetanide $1 \mathrm{mg}$; torasemide $20 \mathrm{mg}$ ).

In our patient's population, a new response-to-diuretic (R-to-D) index including both weight changes and diuresis on Day 4 was calculated as follows:

$$
\text { R-to-D }=\left[\left(\mathrm{W}_{\mathrm{d} 4}-\mathrm{W}_{\text {baseline }}\right) / \text { Fdose }\right]+\log _{\mathrm{n}} \text { diuresis }
$$

where $\mathrm{W}_{\mathrm{d} 4}$ is the weight at day 4 (in $\mathrm{kg}$ ), $\mathrm{W}_{\text {baseline }}$ is the weight at baseline, Fdose is the dose of furosemide on Days 1-3 (40 mg) (equivalent doses: bumetanide $1 \mathrm{mg}$; torasemide $20 \mathrm{mg}$ ) and diuresis refers to the diuresis at Day 4 (in liters).

According to the formula, the $\log _{n}$ of a number $<1$ is a negative number allowing an amplification of the effect of a Day-4 diuresis $<1$ L/day [17] which was considered one of the criteria for a safe discharge of HF patients after an acute decompensation together with improvement of NYHA class, heart rate $<100 \mathrm{bpm}$, pulse oximetry in ambient air $>90 \%$, $90<$ systolic blood pressure $<120 \mathrm{mmHg}$ [19].

According to the study protocol, CHF outpatients were checked at 3 and 6 months after discharge. In the event of a worsening of the clinical status (worsening dyspnea, body weight increase or edema, cardiac arrhythmias), a clinical control was provided. The long-term medical therapy associated with loop diuretic included: angiotensin converting enzyme inhibitors (ACE) (enalapril, ramipril), angiotensin receptor blockade (valsartan) in the case of enalapril/ramipril intolerance, beta-blockers (bisoprolol), digoxin and spironolactone at low dose. For beta-blockers, ACE and angiotensin receptor blockade, the patients' maximum tolerated dose was used, after an adequate titration period.

\subsection{Echocardiography Assessment}

Echocardiograms were performed with a Vivid 7 computed sonography system (GE Medical Systems, Waukesha, Wisconsin, USA) according to the recommendations of the American Society of Echocardiography [20]. Two-dimensional apical 2- and 4-chamber views were used for volume measurements; left ventricular ejection fraction (LVEF) was calculated with a modified Simpson's method using biplane apical (2- and 4-chamber) views. All the echo examinations were performed by expert operators blinded to the results of BNP assay; the intra-observer variability in the evaluation of LVEF was found to be $<5 \%$. Echocardiographic measurements including LV end-diastolic diameter and the diastolic thickness of the ventricular septum and the posterior LV wall were determined according to the American Society of Echocardiography recommendations [20]. The tricuspid annular plane systolic excursion (TAPSE) was measured in a four-chamber view by placing the 2D cursor at the tricuspid lateral annulus and measuring the distance of systolic annular RV excursion along a longitudinal line defining the end of systole as the end of the T-wave in the electrocardiogram. Systolic right ventricular (or pulmonary artery) pressure was calculated using the modified Bernoulli equation: $\mathrm{PAP}=4 \times$ (tricuspid systolic jet $)^{2}+10 \mathrm{mmHg}$ (estimated right atrial pressure).

\subsection{Blood Collection}

At the time of presentation, a blood sample was collected into tubes containing ethylene diaminetetracetic acid and blood was immediately processed. The NT-pro-BNP was performed with a commercially available immunoassay (ADVIA Centaur XPT, Siemens Healthcare Diagnostics, Inc. Tarrytown, NY, USA) on an ADVIA Centaur analyzer according to the established methods.

The assay results were complete in $15 \mathrm{~min}$. Performance characteristics of the test: Assay range $35-35,000 \mathrm{pg} / \mathrm{mL}$; Total CV 9.2-11.4\%. 


\subsection{Cardiovascular Events}

Clinical outcome was evaluated in terms of death or recurrent heart failure hospitalization over a 6-month follow-up period. A scheduled outpatient visit or phone contact at 90 and 180 days after discharge was provided. Heart failure recurrence was defined as any hospital admission with a primary or secondary diagnosis of volume overload or low output due to pump failure, acute coronary syndrome complicated by heart failure, ventricular arrhythmia associated with left ventricular dysfunction or heart failure related to WRF. All these events were defined through our results as composite outcome in terms of adverse events/cardiac mortality. The study protocol conforms to the ethical guidelines of the 1975 Declaration of Helsinki, it was registered in Research Registry n.1965 and approved by the Ethical Committee of ASLCN1-ASO Santa Croce (ethical approved number 10-18 September 2018). Written informed consent was obtained from all participants prior to the study.

\subsection{Statistical Analysis}

All data were analyzed with intention-to-treat principles. Continuous variables are expressed as median interquartile range (IQR), while discrete variables are presented as counts with percentages $(\%)$. The Mann-Whitney $U$ test and $\chi^{2}$-test were used as indicated to compare among groups. Spearman rho's correlation coefficient was employed to evaluate the correlation between $\mathrm{R}$ to $\mathrm{D}$ formula and DR formula. The receiving operating characteristics (ROC) curve was employed to evaluate outcome prediction by $\mathrm{R}$ to $\mathrm{D}$ and $\mathrm{DR}$ formulas. The Cox regression analysis was used to assess the independent relationship between quartiles of $R$ to $D$ and quartiles of $D R$ for the composite outcome of rehospitalization or death with adjustment for age, gender, hypertension, diabetes mellitus, coronary artery disease (CAD) and chronic kidney disease (CKD). All statistical tests were two-tailed, with $p$ value $<0.05$ considered significant. All the analyses were performed by using the SPSS 20.0 for Windows (SPSS, IBM, Armonk, NY, USA).

\section{Results}

Two-hundred and sixty-three patients (62\% male, age 78 (70-83) years old) were discharged after a new diagnosis of $\mathrm{CHF}$ or for acute decompensation in chronic/de novo CHF and were requested to enter the study. All patients were treated with furosemide intravenous for acute decompensation; none was treated with torasemide/bumetanide. None had been treated with thiazide diuretics and $110 / 205$ in the no-event group vs. 32/58 in the event group assumed aldosterone antagonist $(p=n s)$. The etiology of HF was interpreted as: $47 \%$ ischemic; $11 \%$ cardiomyopathy; $20 \%$ hypertensive and finally $22 \%$ valvular or others. During the 6-month follow-up 58 (22.05\%) events were scheduled (21 cardiac deaths, 37 rehospitalization for HF) defining the event group. The remaining 188 patients who did not experience any predefined cardiovascular events formed the no-event group.

Patients who experienced a CV-event had worse renal function $(p=0.001)$, higher pro-BNP $(p=0.004)$, lower DR $(p=0.02)$ and R-to-D $(p=0.03)$ (Table 1$)$; not surprisingly, the furosemide dosage on Day 4 proved to be higher in the event group $(p=0.01)$, while neither the amount of fluid output on Day 4 nor the body weight were significantly different in the two groups ( $p=0.2$ and $p=0.56$, respectively).

In all patients median R to D was $1.69(0.97-3.28)$ and median DR was $1.44(0.67-2.80)$. Only R-to-D was inversely related to in-hospital stay $(\mathrm{r}=-0.23 ; p=0.01)$. ROC curve analysis showed that both $\mathrm{DR}$ (area under curve (AUC): $0.39(0.32-0.47) ; p=0.02)$ and R-to-D (AUC: $0.40(0.32-0.48) ; p=0.03$ ) were able to predict poor prognosis at 180 days (Figure 1 ). 
Table 1. Differences between main variables in event group (180 days rehospitalization or death) vs. no-event group.

\begin{tabular}{|c|c|c|c|}
\hline Variables & No-Event Group (205 pts) & Event Group (58 pts) & $p$ Value \\
\hline Age & $77(70-82)$ & $79(76-85)$ & 0.01 \\
\hline Gender Male (\%) & 63 & 64 & 0.88 \\
\hline Sodium (MEq/L) & $140(138-143)$ & $140(136-142)$ & 0.04 \\
\hline Creatinine $(\mathrm{mg} / \mathrm{dL})$ & $1.06(0.82-1.45)$ & $1.53(1.04-2.05)$ & $<0.001$ \\
\hline GFR $\mathrm{mL} / \mathrm{min} / 1.73 \mathrm{mq}$ & $52(40-68)$ & $39(28-50)$ & $<0.001$ \\
\hline $\mathrm{Hb}(\mathrm{gr} / \mathrm{L})$ & $12(10.5-13.7)$ & $12.6(11.2-13.5)$ & 0.22 \\
\hline RDW & $14.8(13.8-16)$ & $15.9(14.7-17.7)$ & $<0.001$ \\
\hline Pro-BNP (pg/mL) & $5210(1851-14,901)$ & $12,922(7074-27,769)$ & $<0.001$ \\
\hline $\operatorname{LVEF}(\%)$ & $47(35-55)$ & $38(26-50)$ & 0.001 \\
\hline $\operatorname{LVESD}(\mathrm{mm})$ & $37(30-46)$ & $45(35-57)$ & 0.008 \\
\hline LVEDD (mm) & $51(46-60)$ & $59(48-66)$ & 0.04 \\
\hline TAPSE (mm) & $17(15-20)$ & $16(15-20)$ & 0.32 \\
\hline PASP (mmHg) & $37(30-45)$ & $45(35-58)$ & 0.003 \\
\hline Length of hospital stay (days) & $10(7-14)$ & $10(7-15)$ & 0.35 \\
\hline Baseline body weight (kg) & $73.9(63-89.3)$ & $70.9(56.8-89.9)$ & 0.30 \\
\hline Body weight at Day $4(\mathrm{~kg})$ & $72.4(60.4-87.8)$ & $70.9(58-87.4)$ & 0.77 \\
\hline Diuresis on Day 4 (mL/die) & $1800(1300-2600)$ & $1900(1400-2800)$ & 0.44 \\
\hline Diuretic dosage on Day 4 (mg/die) & $60(50-100)$ & $100(50-237)$ & 0.005 \\
\hline R-to-D (kg/40 mg furosemide) & $1.74(1.02-3.48)$ & $1.53(0.77-2.49)$ & 0.03 \\
\hline Diuretic response (kg/40 mg furosemide) & $1.50(0.75-3.20)$ & $1.29(0.53-2.17)$ & 0.02 \\
\hline Diabetes mellitus N (\%) & 30 & 50 & 0.004 \\
\hline Coronary artery disease $\mathrm{N}(\%)$ & 39 & 33 & 0.38 \\
\hline Hypertension (\%) & 65 & 81 & 0.03 \\
\hline CKD $(\%)$ & 66 & 83 & 0.01 \\
\hline $\mathrm{AF}(\%)$ & 34 & 53 & 0.02 \\
\hline
\end{tabular}

GFR—glomerular filtration rate; $\mathrm{Hb}$-hemoglobin level; $\mathrm{RDW}$-red dispersion width; pro-BNP—brain natriuretic peptide; LVEF-left ventricular ejection fraction; LVESD—left ventricular end-systolic diameter; LVEDD—left ventricular diastolic diameter; TAPSE — tricuspid annular plane systolic excursion; PASP—pulmonary artery systolic pressure; $\mathrm{R}$-to-D—response to diuretic; $\mathrm{CKD}$ — chronic renal disease; $\mathrm{AF}$ —atrial fibrillation.

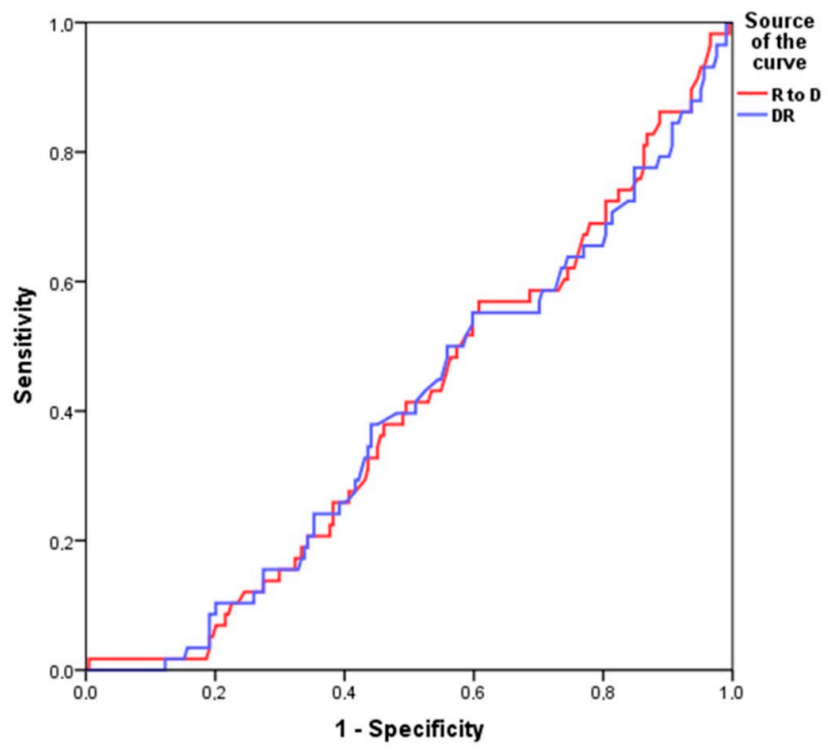

\begin{tabular}{ccc}
\hline Variables & AUC (CI) & $p$ Value \\
\hline DR & $0.39(0.32-0.47)$ & 0.02 \\
R-to-D & $0.40(0.32-0.48)$ & 0.03 \\
\hline
\end{tabular}

Figure 1. Receiving operating characteristics (ROC) curve analysis comparing diuretic response (DR) and response to diuretic (R-to-D). AUC, area under curve; $\mathrm{CI}$, confidence interval. 
Due to significant findings obtained by ROC curve analysis we divided both DR (Q1: DR < 0.67; Q2: $0.67 \leq \mathrm{DR}<1.44 ; \mathrm{Q} 3: 1.44 \leq \mathrm{DR}<2.80$; Q4: $\mathrm{DR} \geq 2.80$ ) and R-to-D (Q1: R-to-D < 0.97; Q2: $0.97 \leq \mathrm{R}$-to-D $<1.69$; Q3: $1.69 \leq \mathrm{R}$-to-D $<3.28$; Q4: R-to-D $\geq 3.28$ ) in quartiles according to median values in all patients. Univariate analysis showed that only Q1 of both DR (HR: 2.56 (1.17-5.63); $p=0.02$ ) and R-to-D (HR: 2.53 (1.11-5.67); $p=0.03$ ) were significantly related to worse outcome. After adjustment for demographic variables and cardiovascular risk factors, multivariable analysis demonstrated that both Q1 (HR: $2.36(1.02-5.47) ; p=0.04)$ and Q2 (HR: $2.52(1.04-6.10) ; p=0.04)$ of R-to-D were related to poor prognosis; conversely only Q1 of DR (HR: $2.27(1.01-5.08) ; p=0.05$ ) was related to poor outcome at 180 days (Table 2).

Table 2. Univariable and multivariable analysis for the composite endpoint of death from all causes (ACM) or heart failure (HF) rehospitalization according to quartiles of $\mathrm{R}$ to $\mathrm{D}(\mathrm{A})$ and quartiles of DR (B).

\begin{tabular}{|c|c|c|c|c|}
\hline \multicolumn{5}{|c|}{ (A) 180-Day HF Rehospitalization or Death } \\
\hline \multirow[b]{2}{*}{ Variable } & \multicolumn{2}{|c|}{ Univariate Analysis } & \multicolumn{2}{|c|}{ Multivariate Analysis ${ }^{1}$} \\
\hline & HR $(95 \% \mathrm{CI})$ & $p$ Value & HR $(95 \% \mathrm{CI})$ & $p$ Value \\
\hline Q1 R to $\mathrm{D}$ ( $\mathrm{R}$ to $\mathrm{D}<0.97 \mathrm{~kg} / 40 \mathrm{mg}$ ) & $\begin{array}{c}2.53 \\
(1.11-5.67) \\
\end{array}$ & 0.03 & $\begin{array}{c}2.36 \\
(1.02-5.47) \\
\end{array}$ & 0.04 \\
\hline $\mathrm{Q} 2 \mathrm{R}$ to $\mathrm{D}(0.97 \leq \mathrm{R}$ to $\mathrm{D}<1.69 \mathrm{~kg} / 40 \mathrm{mg})$ & $\begin{array}{c}2.09 \\
(0.88-4.93)\end{array}$ & 0.09 & $\begin{array}{c}2.52 \\
(1.04-6.10)\end{array}$ & 0.04 \\
\hline Q3 R to $\mathrm{D}(1.69 \leq \mathrm{R}$ to $\mathrm{D}<3.28 \mathrm{~kg} / 40 \mathrm{mg})$ & $\begin{array}{c}2.26 \\
(0.97-5.29)\end{array}$ & 0.06 & $\begin{array}{c}2.35 \\
(0.99-5.55)\end{array}$ & 0.06 \\
\hline
\end{tabular}

\begin{tabular}{ccccc}
\hline Q4 R to D (R to D $\geq 3.28 \mathrm{~kg} / 40 \mathrm{mg})$ & \multicolumn{2}{c}{ Reference } & \multicolumn{2}{c}{ Reference } \\
\hline \multicolumn{4}{c}{ (B) 180-Day } & HF Rehospitalization or Death \\
Q1 DR (DR $<0.67 \mathrm{~kg} / 40 \mathrm{mg})$ & $\begin{array}{c}2.56 \\
(1.17-5.63)\end{array}$ & 0.02 & $\begin{array}{c}2.27 \\
(1.01-5.08)\end{array}$ & 0.05 \\
\hline Q2 DR $(0.67 \leq \mathrm{DR}<1.44 \mathrm{~kg} / 40 \mathrm{mg})$ & $\begin{array}{c}1.54 \\
(0.66-3.61)\end{array}$ & 0.32 & $\begin{array}{c}1.81 \\
(0.76-4.31)\end{array}$ & 0.18 \\
\hline Q3 DR $(1.44 \leq \mathrm{DR}<2.80 \mathrm{~kg} / 40 \mathrm{mg})$ & $\begin{array}{c}2.13 \\
(0.94-4.81)\end{array}$ & 0.07 & $\begin{array}{c}2.23 \\
(0.97-5.12)\end{array}$ & 0.06 \\
\hline Q4 DR (DR $\geq 2.80 \mathrm{~kg} / 40 \mathrm{mg})$ & \multicolumn{2}{c}{ Reference } & Reference
\end{tabular}

Quartiles R to D: Q1. Abbreviations: CI—confidence interval; DR—-diuretic response; HR—hazard ratio. ${ }^{1}$ adjusted

for age, gender and hypertension, diabetes, CAD and CKD.

One-hundred and forty-six patients had HF reduced ejection fraction (HFrEF) and 117 had HF preserved ejection fraction (HFpEF). Patients with HFpEF were younger (76 (68-82) vs. 79 (73-83); $p=0.03)$ and more frequently affected by hypertension $(78 \%$ vs. $62 \% ; p=0.007)$ with respect to patients with HFrEF. Conversely, patients with HFrEF were more frequently male $(71 \%$ vs. $52 \% ; p=0.003)$ and showed higher rate of diabetes ( $40 \%$ vs. $27 \% ; p=0.04)$ and CAD $(47 \%$ vs. $26 \% ; p=0.001)$ than patients with HFpEF (Table 3).

Moreover HFrEF patients showed higher serum levels of creatinine $(1.28(0.94-1.89)$ vs. 1.02 $(0.80-1.25) \mathrm{mg} / \mathrm{dL} ; p<0.001)$ and hemoglobin $(12.9(11.5-14.2)$ vs. $11(10.1-11.9) \mathrm{g} / \mathrm{L} ; p<0.001)$ and lower values of eGFR (45 (32-61) vs. $\left.53(45-73) \mathrm{mL} / \mathrm{min} / \mathrm{m}^{2} ; p<0.001\right)$ with respect to HFpEF patients. ProBNP serum levels were significantly higher in HFrEF than HFpEF patients $(10,285(4027-26,078)$ vs. $3880(1457-10,686) \mathrm{pg} / \mathrm{mL} ; p<0.001)$. Comparing diuretic response formulas in our patients divided according to LVEF. There were higher values of both DR $(1.60(0.72-3.38)$ vs. $1315(0.60-2.24) ; p=0.01)$ and R-to-D (1.95 (1.01-3.75) vs. 1605 (0.94-2.61); $p=0.03)$ in patients with HFpEF with respect to patients with HFrEF (Figure 2). 
Table 3. Baseline characteristics of patients according left ventricular ejection fraction (LVEF).

\begin{tabular}{|c|c|c|c|c|}
\hline \multirow{2}{*}{ Characteristics } & All Patients & HFrEF & HFpEF & \multirow{2}{*}{$p$ Value } \\
\hline & $n=263$ & $n=146$ & $n=117$ & \\
\hline Age (years) & $78(70-83)$ & 79 (73-83) & $76(68-82)$ & 0.03 \\
\hline Gender Male (\%) & 62 & 71 & 52 & 0.003 \\
\hline \multicolumn{5}{|l|}{ Comorbidities (\%): } \\
\hline CAD & 38 & 47 & 26 & 0.001 \\
\hline Diabetes & 34 & 40 & 27 & 0.04 \\
\hline Hypertension & 69 & 62 & 78 & 0.007 \\
\hline $\mathrm{AF}$ & 38 & 41 & 35 & 0.42 \\
\hline CKD & 69 & 74 & 64 & 0.08 \\
\hline \multicolumn{5}{|l|}{ Laboratory variables } \\
\hline Creatinine $(\mathrm{mg} / \mathrm{dL})$ & $1.13(0.85-1.61)$ & $1.28(0.94-1.89)$ & $1.02(0.80-1.25)$ & $<0.001$ \\
\hline eGFR $\left(\mathrm{mL} / \mathrm{min} / \mathrm{m}^{2}\right)$ & $50(37-66)$ & $45(32-61)$ & $53(45-73)$ & $<0.001$ \\
\hline Hemoglobin $(\mathrm{g} / \mathrm{L})$ & $12.1(10.7-13.7)$ & $12.9(11.5-14.2)$ & $11(10.1-11.9)$ & $<0.001$ \\
\hline RDW & $14.9(14-16.4)$ & $15.3(14.2-16.5)$ & $14.6(13.6-16)$ & 0.02 \\
\hline NTproBNP (pg/mL) & $7851(2623-17,989)$ & $10,285(4027-26,078)$ & $3880(1457-10,686)$ & $<0.001$ \\
\hline \multicolumn{5}{|l|}{ Echocardiography: } \\
\hline $\operatorname{EDD}(\mathrm{mm})$ & $53(46-60)$ & $60(51-65)$ & $48(41-53)$ & $<0.001$ \\
\hline $\mathrm{ESD}(\mathrm{mm})$ & $39(31-47)$ & $46(39-55)$ & $32(28-39)$ & $<0.001$ \\
\hline PASP (mmHg) & $40(30-50)$ & $39(30-50)$ & $40(31-50)$ & 0.49 \\
\hline TAPSE (mm) & $17(15-20)$ & $16(15-20)$ & $18(15-20)$ & 0.31 \\
\hline
\end{tabular}

AF-fibrillation; BNP—b-type natriuretic peptide; CKD—chronic kidney disease; EDD—end diastolic diameter; EDS — end systolic diameter; eGFR — estimated glomerular filtration rate; HFpEF—heart failure with preserved ejection fraction; HFrEF - heart failure with reduced ejection fraction; LVEF-Left ventricle ejection fraction; PASP — pulmonary artery systolic pressure; RDW—red cell distribution width; TAPSE—tricuspid annular plane systolic excursion.

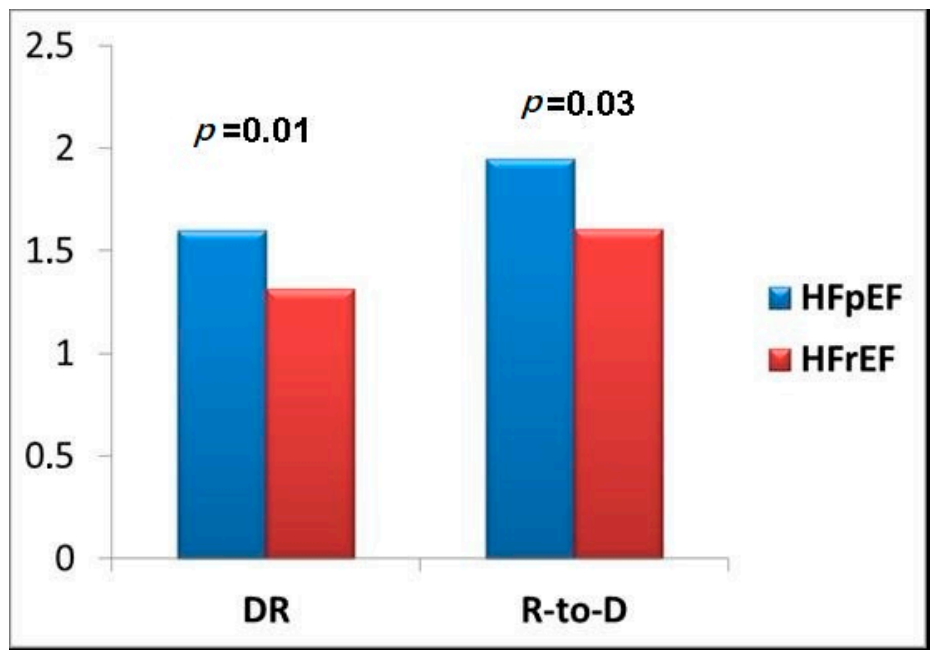

Figure 2. Statistical differences of diuretic response (DR) or response to diuretic (R-to-D) according to the heart failure (HF) classification of reduced/preserved ejection fraction. DR: 1.60 (0.72-3.38) vs. 1315 (0.60-2.24); all patients: 1.44 (0.67-2.80). R to D: 1.95 (1.01-3.75) vs. 1605 (0.94-2.61); all patients: 1.69 (0.97-3.28). HFpEF- heart failure with preserved ejection fraction; HFrEF — heart failure with reduced ejection fraction.

Spearman rho's correlation coefficient showed a strong direct correlation between DR and R-to-D in all patients $(\mathrm{r}=0.93 ; p<0.001)$ and both in $\operatorname{HFrEF}(\mathrm{r}=0.94 ; p<0.001)$ and $\operatorname{HFpEF}(\mathrm{r}=0.91 ; p<0.001)$ (Figure 3). 


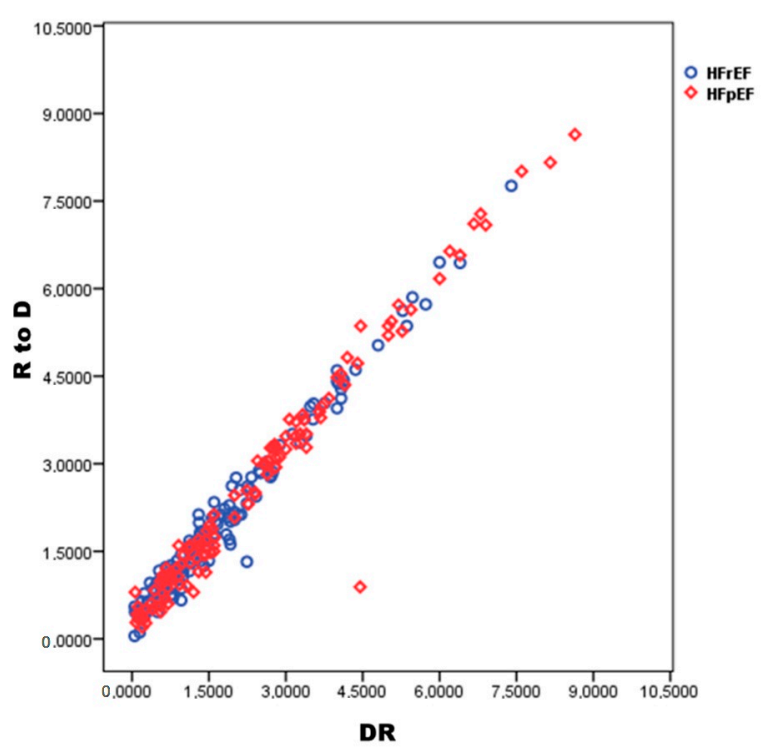

Figure 3. Spearman rho's correlation between diuretic response (DR) or response to diuretic (R-to-D) in the entire population (263 pts) and both in HFrEF or HFpEF.

\section{Discussion}

The most common indicators of diuretic response are net fluid output and changes in body weight. Indeed, body weight reduction does not necessarily imply a consequent decrease in fluid overload [21], but it may be related to an external condition such as sarcopenia cachexia or simple reduction of fluid intake. Recently, a position statement of Heart Failure Association from ESC underlined the utilization of diuretics towards stepped pharmacological diuretic strategies based on diuretic response and common adverse effects (electrolyte disturbances and worsening renal function) [22].

This single-center clinical experience demonstrated that a formula including diuresis together with weight loss during loop diuretic therapy in acute decompensation HF patients seemed to satisfactorily define the clinical outcome after discharge. In CHF patients coming from the PROTECT Trial, Valente et al. [16] calculated the DR as the weight loss per a unitary dose of furosemide (40 mg). A poor diuretic response was independently correlated to low systolic blood pressure, high blood urea nitrogen and predicted 1- and 6-month mortality and cardiovascular hospitalization [16]. Nevertheless, the diuretic responsiveness (diuretic efficiency) has been proposed by calculating the amount of urine produced by the dose of administered loop diuretics [23]. Testani et al. [23] analyzed whether the net fluid output produced per $40 \mathrm{mg}$ furosemide equivalent, may be predictive of clinical outcome in two different populations (coming from the Penn database or from the ESCAPE Trial) admitted for acute decompensation. Dividing into "low" and "high" diuretic efficiency the HF population, they observed that the low diuretic efficiency was correlated to a worsened survival even after adjusting for the diuretic dosage, fluid output and worsening renal function. Unfortunately, despite a persistent increased volume of diuresis after starting a diuretic therapy, a reduction of renal sodium output emerged from the analysis of hypotonic urine diminishing the final objective of the diuretic therapy (to ride the body of excessive sodium). This could explain the interest in dosing the urinary sodium content [24] or only a spot urine sample 1-2 $\mathrm{h}$ following loop diuretic administration [25].

In our clinical experience, DR and R-to-D depicted an equivalent ROC curve analysis (AUC 0.39 and 0.40 , respectively), although the formula R-to-D, through the inclusion of $\log _{n}$ of diuresis on Day 4 to weight loss, proved to better stratify clinical prognosis in alive HF patients discharged after an acute decompensation demonstrating that the lower quartiles $(<1.69 \mathrm{~kg} / 40 \mathrm{mg})$ were significantly correlated to the adverse 6-month prognosis after discharge $(p=0.04)$. In fact, HF patients in whom the R-to-D proved to be $<1.69 \mathrm{~kg} / 40 \mathrm{mg}$ had an HR of 2.5 for developing an events in the follow-up, while for DR (not including the diuresis in the formula) the HR of 2.2 emerged only in the most diuretic 
resistance quartiles $(<0.67 \mathrm{~kg} / 40 \mathrm{mg})$ being not sensitive in case of moderate-light degree of diuretic resistance. Moreover, at univariate analysis, only R-to-D was inversely related to in-hospital stay $(\mathrm{r}=-0.23 ; p=0.01)$. Elderly patients with chronic HF represent most subjects $(70 \%)$ admitted to the hospitals for acute cardiac decompensation; the length of hospitalization usually lasts $>2$ weeks in geriatric wards and readmission is frequent [26]. Advanced HF patients constitute a challenge for cardiologists owing their high mortality and rehospitalization rate $[27,28]$. Therefore, a strategy for stratify planning a tailored clinical follow-up in those patients seems to be mandatory.

The analysis of diuretic resistance in HFrEF and HFpEF demonstrated as DR and R-to-D were significantly higher in HFpEF in comparison to HFrEF ( $p=0.01$ and $p=0.03$, respectively). This result seemed to be correlated to the worst renal function of HFrEF patients in comparison with HFpEF $(p=0.001$ for creatinine and eGFR). The diuretic response has been demonstrated to be strictly correlated to renal impairment $[16,23]$ and it has been shown that HFpEF had a better renal function in comparison to HFrEF [29]. In our clinical experience we demonstrated as the response to loop diuretic in HFrEF patients, measured with DR or R-to-D, was impaired in comparison to HFpEF, adding to our knowledge of the differences between the two different HF syndromes. Indeed, diuretic resistance in HErEF together with older age, comorbidities and worse renal function may play a significant role in explaining the unfavorable post-discharge prognosis in comparison to HFpEF patients. Therefore, HFrEF and HFpEF patients discharged alive after an acute decompensated heart failure (ADHF) with an R-to-D in the lower quartiles should be considered at higher risk of rehospitalization or death.

\section{Conclusions}

In the growing number of parameters used to predict adverse prognosis in HF patients, the diuretic resistance should be considered sensitive and inexpensive. Although adding the computation of diuresis to DR gained similar AUC, the R-to-D formula proved to be more accurate in predicting 6-month adverse events in less severe degree of diuretic resistance (lower quartiles) than DR. Only R-to-D was inversely related to in-hospital stay $(\mathrm{r}=-0.23 ; p=0.01)$. Finally, HFrEF patients seemed to respond worst to loop diuretic according to an impaired renal function in comparison to HFpEF subjects.

\section{Limitations}

We did not evaluate renal function by using specific biomarkers, and we cannot assert whether WRF was related to the progressive deterioration specific to HF patients or effective tubular function impairment. A serial analysis of renal function trajectories after discharge is lacking and it may be useful to better understand underlying mechanism of kidney deterioration and diuresis reduction.

Author Contributions: M.F.: conceptualization, writing-review \& editing; A.R.: methodology; M.T.: data curation; C.F.: data curation; A.P.: writing-review \& editing; G.P.: methodology; G.R.: formal analysis; writing-review \& editing. All authors have read and agreed to the published version of the manuscript.

Funding: This research received no external funding.

Conflicts of Interest: The authors declare no conflict of interest.

\section{References}

1. Ponikowski, P.; Voors, A.A.; Anker, S.D.; Bueno, H.; Cleland, J.G.F.; Coats, A.J.S.; Falk, V.; González-Juanatey, J.R.; Harjola, V.P.; Jankowska, E.A.; et al. 2016 ESC Guidelines for the diagnosis and treatment of acute and chronic heart failure: The Task Force for the Diagnosis and Treatment of acute and of chronic Heart Failure of the European Society of Cardiology with the special contribution of the Heart Failure Association. Eur. Heart J. 2016, 37, 2129-2200. [PubMed]

2. Fonarow, G.C.; Adams, K.F.; Abraham, W.T.; Yancy, C.W.; Boscardin, W.J. Risk Stratification for In-Hospital Mortality in Acutely Decompensated Heart Failure. JAMA 2005, 293, 572-580. [CrossRef] [PubMed]

3. Boland, D.G.; Abraham, W.T. Natriuretic peptides in heart failure. Congest. Heart Fail 1998, 4, $23-33$. 
4. McDonagh, T.A.; Robb, S.D.; Murdoch, D.R.; Morton, J.J.; Ford, I.; Morrison, C.E.; Tunsell-Podoe, H.; McMurray, J.J.; Dargie, H.J. Biochemical detection of left-ventricular systolic dysfunction. Lancet 1998, 351, 9-13. [CrossRef]

5. Maisel, A.S.; Krishnaswamy, P.; Nowak, R.M.; McCord, J.; Hollander, J.; Duc, P.; Omland, T.; Storrow, A.B.; Abraham, W.T.; Wu, A.H.B.; et al. Rapid measurement of B-type natriuretic peptide in the emergency diagnosis of heart failure. N. Engl. J. Med. 2002, 347, 161-167. [CrossRef]

6. Berger, R.; Huelsman, M.; Strecker, K.; Bojic, A.; Moser, P.; Stanek, B.; Pacher, R. B-type Natriuretic Peptide predicts sudden death in patients with chronic heart failure. Circulation 2002, 105, 2392-2397. [CrossRef]

7. Feola, M.; Aspromonte, N.; Canali, C.; Ceci, V.; Giovinazzo, P.; Milani, L.; Quarta, G.; Ricci, R.; Scardovi, A.B.; Uslenghi, E.; et al. Prognostic value of plasma brain natriuretic peptide, urea nitrogen and creatinine in outpatients $>70$ years of age with heart failure. Am. J. Cardiol. 2005, 96, 705-709. [CrossRef]

8. Van Kimmenade, R.R.; Jannuzzi, J.L.; Ellinor, P.T.; Sharma, U.C.; Bakker, J.A.; Low, A.F.; Martinez, A.; Crijns, H.J.; MacRae, C.A.; Menhereere, P.; et al. Utility of amino-terminal Pro-Brain Natriuretic Peptide, Galectin-3 and Apelin for the evaluation of patients with acute heart failure. J. Am. Coll. Cardiol. 2006, 48, 1217-1224. [CrossRef]

9. Neuberg, G.W.; Miller, A.B.; O'Connor, C.M.; Belkin, R.N.; Carson, P.E.; Cropp, A.B.; Frid, D.J.; Nye, R.G.; Pressler, M.L.; Wertheimer, J.H.; et al. Diuretic resistance predicts mortality in patients with advanced heart failure. Am. Heart J. 2002, 144, 31-38. [CrossRef]

10. Ellison, D.H. Diuretic therapy and resistance in congestive heart failure. Cardiology 2001, 96, $132-143$. [CrossRef]

11. Sambandam, K.K. Effective use of loop diuretics in heart failure exacerbation: A Nephrologist's view. Am. J. Med. Sci. 2014, 347, 139-145. [CrossRef] [PubMed]

12. Cowie, M.R.; Komajda, M.; Murray-Thomas, T.; Underwood, J.; Ticho, B. Prevalence and impact of worsening renal function in patients hospitalized with decompensated heart failure: Results of prospective outcomes study in heart failure (POSH). Eur. Heart J. 2006, 27, 1216-1222. [CrossRef] [PubMed]

13. Forman, D.E.; Butler, J.; Wang, Y.; Abraham, W.T.; O'Connor, C.M.; Gottlieb, S.S. Incidence, predictors at admission, and impact of worsening renal function among patients hospitalized with heart failure. J. Am. Coll. Cardiol. 2004, 43, 61-67. [CrossRef] [PubMed]

14. Aronsonk, D.; Burger, A.J. The relationship between transient and persistent worsening renal function and mortality in patients with acute decompensated heart failure. J. Card. Fail 2010, 16, 541-547. [CrossRef] [PubMed]

15. Givertz, M.M.; Postmus, D.; Hillege, H.L.; Mansoon, G.A.; Massie, B.M.; Davison, B.A.; Ponikowski, P.; Metra, M.; Teerlink, J.R.; Cleland, J.G.; et al. Renal function trajectories and clinical outcomes in acute heart failure. Circ. Heart Fail 2014, 7, 59-67. [CrossRef]

16. Valente, M.A.E.; Voors, A.A.; Damman, K.; Van Veldhuisen, D.J.; Massie, B.M.; O'Connor, C.M.; Metra, M.; Ponikowski, P.; Teerlink, J.R.; Cotter, G.; et al. Diuretic response in acute heart failure: Clinical characteristics and prognostic significance. Eur. Heart J. 2014, 35, 1284-1293. [CrossRef]

17. Feola, M.; Testa, M.; Ferreri, C.; Cardone, M.; Sola, M.; Ariotti, S.; Rosso, G.L. Role of Response-to-Diuretic in predicting prognosis in discharged heart failure patients after an acute decompensation. Arch. Med. Res. 2018, 49, 198-204. [CrossRef]

18. Filippatos, G.; Parissis, J.T. Heart failure diagnosis and prognosis in the elderly: The proof of the pudding is in the eating. Eur. J. Heart Fail 2011, 13, 467-471. [CrossRef]

19. Peacock, W.F. Rapid optimization: Strategies for optimal care of decompensated congestive heart failure patients in the emergency department. Rev. Cardiovasc. Med. 2003, 3, 41-48.

20. Sahn, D.J.; Demaria, A.; Kisslo, J.; Weyman, A. The committee on M mode standardisation of the American Society of Echocardiography: Recommendations regarding quantification in M-mode echocardiography: Results of a survey of echocardiographic measurements. Circulation 1978, 58, 1072-1083. [CrossRef]

21. Testani, J.M.; Brisco, M.A.; Kociol, R.D.; Jacoby, D.; Bellmkonda, L.; Parikh, C.R.; Coca, S.G.; Tang, W.H. Substantially discrepancy between fluid and weight loss during acute decompensated heart failure treatment. Am. J. Med. 2015, 128, 776-783. [CrossRef] [PubMed]

22. Mullens, W.; Dammman, K.; Harjola, V.P.; Mebazaa, A.; La Rocca, H.P.B.; Martens, P.; Testani, J.M.; Tang, W.H.W.; Orso, F.; Rossignol, P.; et al. The use of diuretics in heart failure wit congestion-a position 
statement from the Heart Failure Association of the European Society of Cardiology. Eur. J. Heart Fail 2019, 21, 137-155. [CrossRef]

23. Testani, J.M.; Brisco, M.A.; Turner, J.M.; Stpatz, E.S.; Bellumkonda, L.; Parikh, C.R.; Tang, W.W. Loop diuretic efficiency: A metric of diuretic responsiveness with prognostic importance in acute decompensated heart failure. Circ. Heart Fail 2014, 7, 261-270. [CrossRef] [PubMed]

24. Singh, D.; Shrestha, K.; Testani, J.M.; Verbrugge, F.H.; Dupont, M.; Mullens, W.; Tang, W.H. Insufficient natriuretic response to continous intravenous furosemide is associated with poor long-term outcomes in acute decompensated heart failure. J. Card. Fail 2014, 20, 392-399. [CrossRef] [PubMed]

25. Testani, J.M.; Hanberg, J.S.; Cheng, S.; Rao, V.; Onyebeke, C.; Laur, O.; Kula, A.; Chen, M.; Wilson, F.P.; Darlington, A.; et al. Rapid and highly accurate prediction of poor diuretic natriuretic response in patients with heart failure. Circ. Heart Fail 2016, 9, e002370. [CrossRef]

26. Di Lenarda, A.; Scherillo, M.; Maggioni, A.P.; Acquarone, N.; Ambrosio, G.B.; Anniccharico, M.; Bellis, P.; Bellotti, P.; DeMaria, R.; Lavecchia, R.; et al. Current presentation and management of heart failure in cardiology and internal medicine hospital units: A tale of words. The TEMISTOCLE study. Am. Heart J. 2003, 146, 735. [CrossRef]

27. Hernandez, H.F.; Greiner, M.A.; Fonarow, G.C.; Hammill, B.G.; Heidenreich, P.A.; Yancy, C.W.; Peterson, E.D.; Curtis, L.H. Relationship between early physician follow-up and 30-day readmission among Medicare beneficiaries hospitalized for heart failure. JAMA 2010, 303, 1716-1722. [CrossRef]

28. Di Tano, G.; De Maria, R.; Gonzini, L.; Aspromonte, N.; Di Lenarda, A.; Feola, M.; Marini, M.; Milli, M.; Misuraca, G.; Mortara, A.; et al. The 30-day metric in acute heart failure revisited: Data from IN-HF Outcome, an Italian nationwide cardiology registry. Eur. J. Heart Fail 2015, 17, 1032-1041. [CrossRef]

29. Ergatoudes, C.; Schaufelberger, M.; Pivodic, A.; Dahlstrom, U.; Fu, M. Non-cardiac comorbidities and mortality in patient with heart failure with reduced vs preserved ejection fraction. A study using the Swedish heart Failure Registry. Clin. Res. Cardiol. 2019, 108, 1025-1033. [CrossRef]

(C) 2020 by the authors. Licensee MDPI, Basel, Switzerland. This article is an open access article distributed under the terms and conditions of the Creative Commons Attribution (CC BY) license (http://creativecommons.org/licenses/by/4.0/). 